The Astrophysical JouRNAL, 544:204-217, 2000 November 20

(C) 2000. The American Astronomical Society. All rights reserved. Printed in U.S.A.

\title{
ROTATING HALOS AND SPIRALS IN LOW SURFACE BRIGHTNESS GALAXIES
}

\author{
TZIHONG CHIUEH ${ }^{1,2}$ AND YAO-HuAN TsENG ${ }^{3}$ \\ Received 1999 November 30; accepted 2000 April 24
}

\begin{abstract}
As the angular momentum of visible matter in a spiral galaxy is derived from the host cloud, the dark matter halo left behind in the cloud is likely to be in rotation also. In the merging model of galaxy formation, it is shown that the conservation of mass and angular momentum yields a spin parameter within the halo increasing toward the core as $r^{-1 / 3}$ for an isothermal halo and as $r^{-1 / 2}$ for a halo with the universal density profile. Hence, almost corotation between the halo and disk can become possible in the inner galaxy for a relatively high angular momentum halo. Much like normal galaxies, the high angular momentum, low surface brightness galaxies have been observed to exhibit a wide variety of spiral patterns. In this work we explore a mechanism by which disk density waves are excited by corotation resonance between disk and halo, even in disks with a Toomre $Q$ exceeding unity by a sizable margin. Apart from the resonant condition, the effectiveness of disk-halo coupling is found to be determined also by the halo scale height, and enhanced excitation of disk density waves occurs only when the halo scale height is sufficiently small. The spiral-arm pitch angles depend on the halo scale height as well, with the more loosely wound arms associated with smaller halo scale height.
\end{abstract}

Subject headings: galaxies: formation — galaxies: halos — galaxies: peculiar — galaxies: spiral

\section{INTRODUCTION}

Recent progress in the study of galaxy formation has centered around the origin of the Hubble sequence. Among all factors, angular momentum has long been suspected to be the key one that determines galaxy morphology. The theoretical issues under intense study along this direction address the following two problems: (1) the acquisition of angular momenta from primordial density fluctuations and (2) the redistribution of angular momentum and mass through galaxy merging (Heavens \& Peacock 1988; Catelan \& Theuns 1997; Heyl, Hernquist, \& Spergel 1996; Dalcanton, Spergel, \& Summers 1997; Jimenez et al. 1997; Mo, Mao, \& White 1998; Mao \& Mo 1998; Nagashima \& Gouda 1998).

Tidal torque acting on a precollapse cloud has been suggested as a viable means for acquiring the initial angular momentum of a galaxy (White 1984). Such an angular momentum acquisition phase continues until the cloud becomes detached from the background Hubble expansion and collapses (Heavens \& Peacock 1988; Catelan \& Theuns 1997). On the other hand, the currently prevailing model of hierarchical structure formation in a cold dark matter universe suggests that the merging of bound objects could also significantly contribute to galaxy angular momentum in the late phase after the cloud has collapsed (Peebles 1969; White 1984; Barnes \& Efstathiou 1987; Heyl et al. 1996; Nagashima \& Gouda 1998). In this hierarchical model, the different orientations of angular momenta associated with the merging orbits can yield a large scatter in the angular momentum distribution of galaxies, thereby accounting for the wide range of galaxy morphology (Dalcanton et al.

\footnotetext{
${ }^{1}$ Physics Department, National Taiwan University, Taipei, Taiwan; chiuehth@phys.ntu.edu.tw.

${ }^{2}$ Institute of Astronomy and Astrophysics, Academia Sinica, Taipei, Taiwan.

${ }^{3}$ Institute of Physics and Astronomy, National Central University, Chung-Li, Taiwan.
}

1997; Jimenez et al. 1997; Mo et al. 1998; Mao \& Mo 1998). An interesting issue naturally arises concerning how the halo gas could eventually become settled into a rotating disk which exhibits the characteristic exponential light distribution and flat rotational curve detected in spiral galaxies. This issue has recently been investigated, and the Tully-Fisher relation has been used in an attempt to find an explanation within the merging model (Mo et al. 1998). An emerging opinion derived from these recent studies is that there should exist a nonnegligible population of low surface brightness (LSB) galaxies associated with large disk angular momentum (O'Nell, Bothun, \& Cornell 1997; Schneider \& Schombert 2000; Bouwens \& Silk 2000). This peculiar population of faint galaxies remains to be uncovered by direct imaging; they may have already been detected and manifested as quasar Ly $\alpha$ absorbing clouds (Mo et al. 1998).

Since an LSB galaxy generally has a low disk density, it can serve as an ideal probe for the investigation of halo mass distribution through the disk rotation curve (van den Bosch et al. 1999; Dalcanton \& Bernstein 2000; Swaters, Madore, \& Trewhella 2000). Interestingly, those LSB galaxies that have already been imaged clearly exhibit a large variety of spiral patterns (McGaugh 1992). As LSB galaxies are thought to possess a high disk angular momentum with a relatively large disk scale length, both factors can only result in a relatively large Toomre $Q$, likely exceeding a value of 2 . In addition, these galaxies are often spatially isolated, with no clear evidence of interactions with other galaxies (Mo, McGaugh, \& Bothun 1994). Hence, the spiral waves are expected to be suppressed in these galaxies, contrary to what has been observed. It is well known that swing amplification can be the most powerful wave amplification mechanism in a differentially rotating and self-gravitating disk (Goldreich \& Lynden-Bell 1965; Julian \& Toomre 1966; Toomre 1981). However, as will be shown later, the swing amplifier ceases to be operative for values of $Q$ beyond $\sqrt{3}$ in a flat rotation gaseous disk. A stellar disk is somewhat more stable than a gaseous disk, and hence its 
critical value of Toomre $Q$ should be somewhat smaller than $\sqrt{3}$. The work of Morozov (1980) shows that the marginally unstable $Q$ is $\sqrt{2}$ for a flat rotation stellar disk.

Despite the finding that spiral arms in LSB galaxies may be due to selection effects, the arm pitch angles do vary over a sizable range, much like those high surface brightness galaxies in different phases of the Hubble sequence. This suggests that some factors other than the disk $Q$-value are perhaps responsible for the excitation of spiral arms in these high angular momentum galaxies. With the recognition of dark halos in rotation, it has recently been proposed that the misalignment of angular momenta between halo and disk can produce warps in the galaxy (Debattista \& Sellwood 1999; Jiang \& Binney 1999; Binney, Jiang, \& Dutta 1998). However, the warps do not contribute to the density contrast to the leading order. In the present work, we wish to point out a different mechanism for producing the spiral density waves: excitation by the corotation resonance between halo and disk in high angular momentum galaxies.

We will show that the efficiency of coupling between disk and halo in disk-halo corotation depends mainly on one important factor: the local vertical scale height of the halo near the disk. When the halo scale height is small, the coupling can be rather efficient. In fact, the halo scale height is to some degree correlated with the halo angular momentum. A rapidly rotating halo tends to be of oblate shape, hence having a relatively small scale height. Such a tendency thus favors the high angular momentum halo exciting disk density waves through the corotation resonance. Notice that oblate halo potentials have already been reported in some galaxies through polar rings (Sackett et al. 1994; Sackett \& Sparke 1990) and gas-disk flaring (Olling 1995, 1996), though it is not yet clear whether the flattened halo is caused by rotation. However, some observational evidence indicates that the inner halos in spirals may be as oblate as $c / a=0.5$, where $c$ and $a$ are the short and long axes of an ellipsoid, and Tremaine \& Ostriker (1999) have proposed that the flattened halo is actually caused by halo rotation arising from disk dragging.

In the present work, we will instead study the dynamics of the disk density waves, given the presence of a rotating halo. The marginally unstable/amplifying condition for spiral density waves is shown to be substantially modified by the presence of a live inner halo which is corotating with some portion of the disk. It should also be mentioned that an unstable system generally has a tendency to adjust itself to a marginally unstable state. A disk containing unstable/ amplified spiral density waves is thus expected to relax to a marginally unstable/amplifying state, by heating the stars that drive the disk to reach a marginally stable configuration. This saturation mechanism may offer a possibility where, by equating the marginally stable Toomre $Q$ to the observed $Q$, one may hope to extract some information about the halo dark matter that helps excite the density waves.

In the analysis to follow, a gaseous shearing-sheet configuration will be assumed in order to simplify the analysis and to illustrate the underlying physics. We envision that the halo dark matter consists of collisionless particles whose velocity dispersion is sufficiently large that they extend vertically over a sizable distance beyond the disk. It should be stressed that, due to the simplification pertaining to the local shearing-sheet approximation, the result of this local analysis can only be taken as an indication of the tendency which the halo response may produce. Quantitative comparisons with observations must await a global analysis including a realistic disk, bulge, and halo.

This paper is organized as follows: We first address the angular momentum distribution of the galaxy halo in $\S 2$, under the scenario of hierarchical galaxy formation. In $\S 3$ we present an analysis of disk density waves with a rigid halo and of those with a live, corotating halo. Section 4 contains results of the $\mathrm{N}$-body simulations, which examine the nonlinear developments of these cases. Section 5 presents the discussion and conclusions. The Appendix gives the condition under which the adiabatic halo assumption adopted to derive our main results is valid.

\section{ANGULAR MOMENTUM DISTRIBUTION IN THE DARK HALO}

We adopt the hierarchical scenario of structure formation, where merging is the primary mechanism for producing present-day galaxies. It has been argued that the merging processes are more likely of cannibalism than of fusion of equal masses (Hausman \& Ostriker 1978). This picture is adopted here. We shall further idealize the collisionless particles as an ideal gas for the evaluation of the angular momentum distribution. This approximation can be a reasonable one if violent relaxation takes place vigorously during the collapse, so that the momentum relaxation mean free path is short compared with the distance from the galaxy center. Let $M(r)$ be the average mass interior of a radius $r$ of a cloud. We may divide the cloud into spherical shells of finite thickness, with each shell containing a smaller mass $\Delta M$ of equal magnitude. Each $\Delta M$ is added to the primary galaxy at different epochs of the galaxy merging history by capture; the magnitude of angular momentum associated with each $\Delta M$ is assumed to be the same.

The galaxy spin parameter, $\lambda_{\text {gal }} \equiv\left|J_{\text {gal }}^{2} E_{\text {gal }}\right|^{1 / 2} / G M_{\text {gal }}^{5 / 2}$, is a measure of the relative importance of rotational support compared to gravity, where $J_{\text {gal }}$ is the galaxy angular momentum and $E$ the binding energy. It has been shown by numerical simulations that a global halo spin parameter $\lambda_{\text {gal }}$ as large as 0.1 is not uncommon (Nagashima \& Gouda 1998; Cole \& Lacey 1996; Steinmetz \& Bartelmann 1995; Warren et al. 1992). It is in this regime of high global spin parameter, $\lambda_{\text {gal }} \geq 0.1$, that we are most interested.

In an ideal fluid, the frozen-in condition of vortex lines demands that the vorticity field $\omega$ is conserved, where $\omega=$ $\nabla \times v$. The specific angular momentum is no more than the circulation of vortex motion, which amounts to $\int \omega \cdot d \boldsymbol{A}$, with $d \boldsymbol{A}$ being the surface area into which the vortex lines penetrate. When matter of a constant specific angular momentum accretes in the directions perpendicular to the vortex lines, both circulation and mass should increase in proportion. However, matter may also accrete in the direction along the vortex lines, in which case the circulation remains unchanged but the mass increases. We conclude from the above considerations that the accumulated specific angular momentum $j(r)$ within a radius $r$ scales as $j(r)^{3 / 2} \propto M(r)$ and therefore the accumulated angular momentum scales as $J \propto M^{5 / 3}$. However, the vorticity is a vector field, and the accumulated angular momentum is a net result of cancellation among the acquired angular momenta of different orientations. Nevertheless, the high $\lambda_{\text {gal }}$ galaxies are likely to be biased toward situating in a large-scale environment that has a net angular momentum. If indeed so, the angular momentum of the merging orbit 
has a small but finite mean, and the scaling relation between the net local angular momentum $\bar{J}(r)$ and mass remains the same, i.e.,

$$
\bar{J}(r)=c_{\text {gal }} M^{5 / 3}(r),
$$

where $c_{\text {gal }}$ is a constant within a single galaxy but varies from one galaxy to another. Evidence of this scaling relation has been provided by recent galaxy merging simulations which aim to investigate the angular momentum distribution in a collection of galaxies (Nagashima \& Gouda 1998). Since the proportional constant $c_{\mathrm{gal}}$ of equation (1) is a random variable, this simulation work does show that the random distribution of galaxy spin parameter $\lambda_{\text {gal }}$ indeed traces the randomness of $c_{\text {gal }}$.

We may thus define an interior spin parameter $\lambda_{\text {in }}(r) \equiv$ $\left|\bar{J}^{2}(r) E(r)\right|^{1 / 2} / G M^{5 / 2}(r)$. Substituting the mass-angular momentum scaling relation into the spin parameter, we find that $\lambda_{\text {in }}(r) \propto\left[M(r)^{1 / 3} / r\right]^{1 / 2}$. For an isothermal halo, where $M \propto r$, we have $\lambda_{\text {in }}(r) \propto r^{-1 / 3}$ and the halo rotation speed $V_{c} \propto r^{-1 / 3}$. Both increase toward the galactic center before reaching the isothermal core at $r=r_{c}$. Inside the isothermal core, the halo rotation is rigid body with $\lambda_{\text {in }} \sim$ const and $V_{c} \propto r$. If we instead replace the isothermal density distribution by the "universal" density profile, i.e., $\rho \propto$ $\left[r\left(r+r_{c}\right)^{2}\right]^{-1}$ (Navarro, Frenk, \& White 1995, 1997), the spin parameter also increases inward as $\lambda_{\text {in }} \propto r^{-1 / 2}$ for $r>$ $r_{c}$ and $\lambda_{\text {in }} \propto r^{-1 / 6}$ for $r<r_{c}$. Of particular relevance is that $\lambda_{\text {in }}(r)$ increases inward. It indicates that the rotational support of the halo becomes more pronounced near the halo core, e.g., $r_{c} \sim 5 \mathrm{kpc}$ for LSB galaxies (Swaters et al. 2000 ), and the interior spin parameter may increase by a factor of a few from its global value.

To give an estimate, we take a high global spin parameter $\lambda=0.15$ on the scale of $100 \mathrm{kpc}$ for a typical LSB galaxy. The isothermal model gives $\lambda_{\text {in }}\left(r_{5}\right) \sim 0.4$, where $r_{5}$ stands for the distance of $5 \mathrm{kpc}$. Upon substituting the above scaling relations and integrating the binding energy radially, we find that $V_{\text {halo }}(r) \sim \lambda_{\text {in }}(r)(5 \pi / 12)[2 G M(r) / r]^{1 / 2}$ whereas $V_{\text {disk }}(r)=[G M(r) / r]^{1 / 2}$. Thus, with $\lambda_{\text {in }}\left(r_{5}\right) \sim 0.4$, it yields that $V_{\text {halo }}\left(r_{5}\right) \approx 0.75 V_{\text {disk }}\left(r_{5}\right)$. For the proposed corotation resonance to be operative, we will show later that it requires $\left[1-\left(V_{\text {halo }} / V_{\text {disk }}\right)\right]^{2}<c$, with $c \ll 1$. We take $c=0.2$ to estimate the range of radius within which the corotation coupling becomes effective. From the scalings $V_{\text {halo }} \propto r^{-1 / 3}$ and $V_{\text {disk }} \propto$ const for $r>r_{c} \sim 5 \mathrm{kpc}$, it gives that the outer boundary of the valid corotation region is about $20 \mathrm{kpc}$, a sufficiently large range for wave excitation.

Though it is instructive to examine the extent to which the inner halo may be flattened by rotation, a self-consistent treatment of such an equilibrium is rather involved and beyond the main points this work intends to address. However, as an estimate, by comparing the halo stress in different directions we find that rotation as fast as $V_{\text {halo }} \approx$ $0.75 V_{\text {disk }}$ can give a halo as flat as $c / a \leq 0.5$ inside $r_{c} \sim 5$ $\mathrm{kpc}$, where $a$ and $c$ are the major and minor axes of equidensity contours.

Recently, it has also been pointed out that the clumpy inner halo of a spiral galaxy can be made to rotate quite rapidly (Tremaine \& Ostriker 1999). The spun-up clumpy halo is proposed to be caused by the dragging of galactic disks through dynamical friction. This specific mechanism is, however, operative only over a relatively long timescale and effective only after the disk has been formed. Such a mechanism adds an extra angular momentum to the inner halo in the long run, probably making the halo rotate slightly faster than the above scaling laws suggest.

\section{SPIRAL DENSITY WAVES IN A ROTATING HALO: THEORY}

\subsection{Rigid Halo}

We shall start with a review of the density waves in a rigid halo. It serves to contrast the differences and similarities with a rotating live halo. In the absence of dynamical response from the halo dark matter, the only effect provided by the halo is simply a rigid gravitational potential well, within which the gaseous (or stellar) disk is confined. The background gravitational potential gives rise to an asymptotically flat rotation curve of a disk galaxy. In the shearingsheet approximation, the Cartesian coordinate is adopted to represent the radial $(x)$ and azimuthal $(y)$ positions, and the flat rotation yields the shearing parameter $A(\equiv-R d \Omega /$ $d R)=\Omega$ and the epicyclic frequency $\kappa\left[\equiv(4 \Omega B)^{1 / 2}\right]=$ $\sqrt{2} \Omega$. Here, $R$ is the axial radius, $\Omega$ the local angular velocity of the disk, and $B \equiv \Omega-A / 2$.

We may further use the shearing coordinates $x, \eta(\equiv y-A x t)$ to simplify the analysis, where the disk has been assumed to be infinitely thin. The surface density perturbation of gases in the disk is represented by $\delta \Sigma e^{i\left(k_{x} x+k_{y} \eta\right)}$, where $\delta \Sigma$ has been shown to satisfy (Goldreich \& Tremaine 1978)

$$
\begin{aligned}
& \frac{d}{d \tau}\left[\frac{A^{2}}{1+\tau^{2}} \frac{d}{d \tau}\left(\frac{\delta \Sigma}{\Sigma}\right)\right] \\
& \quad+\left[k_{y}^{2} a^{2}+\frac{4 \Omega B}{1+\tau^{2}}-\frac{4 A B}{\left(1+\tau^{2}\right)^{2}}-\frac{2 \pi G \Sigma k_{y}}{\sqrt{1+\tau^{2}}}\right] \frac{\delta \Sigma}{\Sigma}=0
\end{aligned}
$$

and $\tau \equiv A t+k_{x} / k_{y}$, where $G$ is the gravitational constant, $\Sigma$ the equilibrium surface density, and $a$ the gas sound speed. Note that $\tau$ can be viewed as cot $[\psi(t)]$, where $\psi(t)$ is the instantaneous pitch angle of the spiral arm subtended from the azimuthal direction and hence $\psi=0$ corresponds to an axisymmetric density wave.

Let $\delta \xi \equiv \delta \Sigma / \Sigma\left(1+\tau^{2}\right)^{1 / 2}$, and one may easily show that equation (2) becomes

$$
\left(\frac{d^{2}}{d \tau^{2}}+S_{0}^{2}\right) \delta \xi=0
$$

with

$$
S_{0}^{2} \equiv\left[2-\frac{2 \sqrt{2}}{Q} \frac{k a}{\Omega}+\frac{k^{2} a^{2}}{\Omega^{2}}\right]-\left[\frac{4}{1+\tau^{2}}-\frac{3}{\left(1+\tau^{2}\right)^{2}}\right],
$$

where $k \equiv k_{y}\left(1+\tau^{2}\right)^{1 / 2}, Q \equiv \kappa a / \pi G \Sigma$, and the flat rotation curve has been assumed $(A=\Omega)$. The first bracket in $S_{0}^{2}$ is readily recognized as the dispersion relation for the axisymmetric density wave and the second as the swing amplifier first pointed out by Goldreich \& Lynden-Bell (1965) and shortly thereafter by Julian \& Toomre (1966).

When $S_{0}^{2}<0$, the swing amplifier can effectively enhance the seed density perturbations. It is apparent that when $Q\left(\equiv \kappa a / \pi G \Sigma_{\text {disk }}\right)>1$, the axisymmetric perturbations become stable. The marginal stability for the swing amplifier can be found by minimizing equation (4) with respect to both $k a / \Omega$ and $\left(1+\tau^{2}\right)^{-1}$. A straightforward calculation shows that for a flat rotation curve the critical value $Q_{c}=$ 
$\sqrt{3}$ and the minimum of $S_{0}^{2}$ occurs at $k_{y} a / \Omega=\sqrt{2 / 3}$ and $\tau=1 / \sqrt{2}$, i.e., an arm pitch angle of $\psi=\cot ^{-1} \sqrt{1 / 2} \approx$ 54.7 from the azimuthal direction. (It should be emphasize that the actual arm pitch angle at the maximum amplitude should be substantially less than 54.7 since the maximum amplitude occurs well after the wave front passes through the phase of maximum growth rate.)

In addition to the above simplest version of swing amplification, a kinetic theory has been examined to determine the critical $Q$-value of a stellar disk, and it has been reported that $Q_{c}=\sqrt{2}$ (Morozov 1980). Effects of finite vertical thickness have also been studied in the past (Yue 1982), and they tend to stabilize the disk. For axisymmetric perturbations in a finite-thickness disk, the marginally stable $Q$ becomes less than unity, reduced by a factor $[1+O(\Delta z \kappa)$ a)], where $\Delta z$ is the disk thickness. These factors all point to a conclusion that additional destabilizing mechanisms are indeed needed in order to account for the spiral patterns in LSB galaxies with a large $Q$-value $(>2)$. Moreover, in the inner LSB galaxy, the disk rotation speed gradually declines to rigid-body rotation, i.e., $A \rightarrow 0$ (Swaters et al. 2000), and the swing amplifier becomes less effective, with the threshold $Q$-value necessarily approaching unity in a thin disk. This further reinforces the need for unconventional destabilizing mechanisms for spiral waves.

To explore how the proposed destabilizing mechanism can be built upon the conventional density wave theory, we shall focus on the flat-rotation region of the LSB galaxy. It represents a limiting case with minimal modification to the conventional density wave theory. In the next subsection, the destabilizing dynamical response of a corotating live halo will be presented.

\subsection{Adiabatic Response of a Corotating Live Halo}

Since the halo has a large velocity dispersion, we assume that the velocity dispersion much exceeds both the phase velocities of the disk density waves and the difference of rotation speed between disk and halo. Hence, the potential perturbations of the halo response can be regarded as adiabatic, in the sense that the halo matter can always adjust itself to a quasi-static equilibrium state against any slow change of the disk potential. For simplicity, we assume that the equilibrium halo density satisfies a local Boltzmann distribution, $\rho_{\mathrm{dm}} \propto \exp \left(-\phi / T_{\mathrm{dm}}\right)$. When subject to small potential perturbations $\left(\delta \phi \ll T_{\mathrm{dm}}\right)$, the halo density becomes $\rho_{\mathrm{dm} 0}+\delta \rho_{\mathrm{dm}} \propto \exp \left[-\left(\phi_{0}+\delta \phi\right) / T_{\mathrm{dm}}\right]$, and hence

$$
\delta \rho_{\mathrm{dm}}=-\rho_{\mathrm{dm} 0}(r) \frac{\delta \phi}{T_{\mathrm{dm}}}
$$

where $\rho_{\mathrm{dm} 0} \propto \exp \left(-\phi_{0} / T_{\mathrm{dm}}\right)$.

One may account for this intuitive result from another perspective. When the halo corotates with the disk, the vertical and horizontal momentum of the halo can be made to decouple. Specifically, one may write the vertical momentum equation for the halo dark matter in the rotating frame as

$$
\frac{\partial \delta w_{\mathrm{dm}}}{\partial t}+A x \frac{\partial \delta w_{\mathrm{dm}}}{\partial y}=-\frac{T_{\mathrm{dm}}}{\rho_{\mathrm{dm}}} \frac{\partial \delta \rho_{\mathrm{dm}}}{\partial z}-\frac{\partial \delta \phi}{\partial z},
$$

where $\delta w_{\mathrm{dm}}$ is the vertical velocity perturbation. Since the halo dark matter is hot and the wave is slow, we may ignore the inertia terms on the left of equation (6) to obtain equation (5). A more rigorous analysis that justifies equation (5) is given in the Appendix, where the limit within which equation (5) may apply becomes clear. It is found in the Appendix that only when the difference of rotation speed between halo and disk is comparable to or greater than the halo velocity dispersion will equation (5) fail to hold.

From equation (5), it follows that Poisson's equation becomes

$$
\left[\nabla^{2}+k_{d}^{2}(r)\right] \delta \phi=[4 \pi G \delta(z)] \delta \Sigma,
$$

where $k_{d}^{2}(r) \equiv 4 \pi G \rho_{\mathrm{dm}}(r) / T_{\mathrm{dm}}$, the inverse halo scale height. Thus, the gravity response function changes from the Laplacian to the Helmholtz type. Using the representation $\delta \Sigma(\tau) e^{i\left(k_{x} x+k_{y} \eta\right)}$, we may reexpress equation (7) as

$$
\left(\frac{\partial^{2}}{\partial z^{2}}+k_{d}^{2}-k^{2}\right) \delta \phi(z, \tau)=[4 \pi G \delta(z)] \delta \Sigma(\tau) .
$$

On the disk $(z=0)$, this yields

$$
\delta \phi(z=0, \tau)=-\frac{2 \pi G}{\sqrt{k^{2}-k_{d}^{2}}} \delta \Sigma(\tau) .
$$

Comparing equations (5) and (9), we note that $\delta \Sigma$ and $\delta \rho_{\mathrm{dm}}$ are in phase, but both of them are $180^{\circ}$ out of phase with the potential perturbation $\delta \phi(z=0)$ in the physically relevant regime where $k^{2}>k_{d}^{2}$.

Following the analysis presented in the previous subsection, it is straightforward to show that in the shearing coordinate, equation (3) remains of the same form but equation (4) changes to

$$
\begin{aligned}
S^{2}= & -\frac{2 \sqrt{2} W q\left(1+\tau^{2}\right)}{Q \sqrt{q^{2}\left(1+\tau^{2}\right)-1}}+W^{2}\left(1+\tau^{2}\right) \\
& -\frac{4}{1+\tau^{2}}+\frac{3}{\left(1+\tau^{2}\right)^{2}},
\end{aligned}
$$

where $q^{2} \equiv k_{y}^{2} / k_{d}^{2}$ and $W \equiv a k_{y} / \Omega$. (As before, the flatrotation case has been considered to demonstrate the minimally required halo effect for the corotation excitation of disk density waves.)

Equation (10) shows that when $q^{2}\left(1+\tau^{2}\right)<1$, the factor $S^{2}$ can become complex and the perturbation $\delta \xi$ is oscillatory and undergoes a growing phase within a finite time interval. This oscillatory growing phase persists even for a large value of $Q$, as long as $k_{y}$ is sufficiently small. However, in a realistic system, there is a lower bound to $k_{y}$ limited by the system horizontal size, i.e., $k_{y} \geq R^{-1}$, where $R$ is the axial distance from the center of the galaxy, and hence the oscillatory growing modes are prohibited for a halo locally satisfying $k_{d}<R^{-1}$. Even when these modes do become unstable, in a collisionless stellar disk, the oscillatory perturbations are prone to suffer from the Landau damping that suppresses the wave growth. However, such a damping is not captured in the above hydrodynamic treatment. Alternatively, there can be another nonoscillatory growing perturbation existing in the regime where $q^{2} \geq 1$ and hence $S^{2}$ is real. The most unstable mode of this type assumes $q^{2}=1+\epsilon$, with a positive $\epsilon \rightarrow 0$. These nonoscillatory unstable modes are the hydrodynamic modes and less likely to get coupled to the Landau damping. These hydrodynamic modes can survive in the nonlinear regime, as will be shown in the numerical simulations given in the next section. 
With this particular choice $q^{2}=1+\epsilon$, it follows that equation (9) becomes

$$
\begin{aligned}
S^{2} \approx 2 & -\frac{2 \sqrt{2} W^{\prime}\left(1+\tau^{2}\right)}{Q \sqrt{\tau^{2}+\epsilon}}+W^{\prime 2}\left(1+\tau^{2}\right) \\
& -\frac{4}{1+\tau^{2}}+\frac{3}{\left(1+\tau^{2}\right)^{2}},
\end{aligned}
$$

where $W^{\prime} \equiv a k_{d} / \Omega$. The most destabilizing second term of equation (11) has a coefficient containing $Q$ with a particular combination $a k_{d} / \Omega Q$. Hence, a larger $k_{d}$ requires a larger $Q$ to attain marginal instability. In fact, an unstable system tends to automatically adjust itself toward such a state as the instability proceeds. In other words, the disk stars can get heated, thereby producing an increasingly higher $Q$-value. On the other hand, for a given $Q$, the perturbations can grow into the nonlinear regime more rapidly as $k_{d}$ increases; therefore, to a lesser degree will these rapidly growing unstable modes be involved with the swing amplification. That is, the spiral waves can quickly saturate nonlinearly at a small $\tau$, which yields a larger spiral-arm pitch angle, i.e., more loosely wound spiral arms. This behavior will also be demonstrated in the $N$-body simulations presented below.

In sum, the above analysis leads to a qualitative conclusion that a smaller halo scale height or larger $k_{d}$ tends to produce not only more open spiral arms but also a larger $Q$, as the stellar disk relaxes to approach the marginally unstable state.

\section{SPIRAL DENSITY WAVES IN A ROTATING HALO: $N$-BODY SIMULATIONS}

We now turn to $N$-body simulations to investigate the nonlinear developments of the halo-assisted instability. The value of $k_{d}$ is taken as a given parameter. We adopt equation (7) for the dark matter density perturbation to determine the "dressed" gravity of a star. The gravitational potential $\phi_{*}$ for a star satisfies

$$
\nabla^{2} \phi_{*}+k_{d}^{2} \phi_{*}=4 \pi G m_{*} \delta^{3}(r),
$$

where $r$ is the distance from the star. The solution $\phi_{*}$ has a simple form:

$$
\phi_{*}=\frac{G m_{*}}{r} \cos \left(k_{d} r\right) .
$$

In obtaining this expression, we have chosen a particular solution to the Helmholtz equation such that when $k_{d} r \ll 1$, the "bare" gravity is recovered. The "dressed" gravitational potential has a form reminiscent of the Debye shielding in a plasma, but with an imaginary charge. In this regard, one may conceive this dressed potential as due to the "imaginary shielding" by the halo dark matter (Binney \& Tremaine 1987).

With the stellar potential given by equation (13), we conduct the direct $N$-body simulations in a twodimensional periodic shearing sheet (Toomre 1981). Three cases are investigated: ( $a$ ) rigid halo, $(b)$ live halo with $k_{d}=$ $\pi / \lambda_{c}$, and (c) live halo with $k_{d}=2 \pi / \lambda_{c}$, where the critical length $\lambda_{c}\left(\equiv 4 \pi^{2} G \Sigma_{\text {disk }} / \kappa^{2}\right)$ is the longest unstable wavelength for the axisymmetric modes (Toomre 1981). (As a reference, in the solar neighborhood the critical wavelength $\lambda_{c}$ is about $10 \mathrm{kpc}$ and the radial scale length of the stellar disk of the Milky Way is about $3.4 \mathrm{kpc}$.) The softening length $r_{\text {soft }}$ is chosen to be $0.1 \lambda_{c}$ for cases $a$ and $b$ and $0.05 \lambda_{c}$ for case $c$ to avoid the occasional hard collisions at close encounters. Case $c$ has a softening length one-half that in case $b$ because we expect that its dominant wavelength will be correspondingly smaller. Furthermore, the computational system size is chosen to be $4 \lambda_{c} \times 6 \lambda_{c}$ for cases $a$ and $b$ and $3 \lambda_{c} \times 4 \lambda_{c}$ for case $c$, with the long side along the streamwise direction, so that on average the softened sphere contains sufficiently many particles to avoid discrete particle effects, namely, 14 particles for all three cases.

Shown in Figures $1 a, 1 b$, and $1 c$ are the sequence of evolution of the disk stars over more than one rotation (one rotation $=628 \Delta t$ ) for three different halo scale heights: $(a)$ $k_{d}^{-1}=\infty,(b) k_{d}^{-1}=\lambda_{c} / \pi$, and $(c) k_{d}^{-1}=\lambda_{c} / 2 \pi$, with the same initial $Q(t=0)=1$.6. The length in Figure 1 is normalized to $\lambda_{c}$. It is evident that the rigid halo case yields nearly marginally unstable density perturbations, whereas the live halo cases produce much more rigorous instabilities. For the rigid halo case, $\cot ^{-1}\left(k_{x} / k_{y}\right)$ is found to be about $25^{\circ}-28^{\circ}$ in the nonlinear regime; this is to be contrasted with the unstable angle $\left(\sim 55^{\circ}\right)$ in the linear regime. For case $b$, the spiral-arm pitch angle is also in the range of $25^{\circ}-28^{\circ}$ in the nonlinear regime. In fact the patterns in the two cases are rather similar, except that case $b$ has larger amplitudes. For case $c$, the arm pitch angle in the nonlinear regime lies in the range of $32^{\circ}-37^{\circ}$.

Shown in Figures $2 a, 2 b$, and $2 c$ are the sequences of density-spectrum evolution in the wavenumber space for the rigid case and the two live halo cases. The power spectrum is constructed by employing a technique where one regards the particle, labeled by $j$, as a discrete $\delta$-function to calculate the density Fourier component, i.e., $\Sigma(\boldsymbol{k})=$ $(1 / N) \sum_{j=1}^{N} \exp \left[i \boldsymbol{k} \cdot \boldsymbol{x}_{j}\right]$, where $N$ is the total particle number. In addition, $k_{y}$ assumes discrete values compatible with the finite boundaries in the $y$-direction, whereas $k_{x}$ assumes continuous values because of the background flow shear. The wavenumber is normalized to $\lambda_{c}^{-1}$. It is interesting to note that some nonlinear fluctuations appear in the form of solitary waves in the $k_{x}$ space. The wave packets die out at a moderately high $k_{x}$, but soon after new wave packets reappear at low $k_{x}$. In other words, the spiral density waves recur. The disappearance of the solitary waves at high $k$ may have little to do with the softening length $r_{\text {soft }}$ introduced in the simulations. Although in cases $a$ and $b$ the waves die out at about $k r_{\text {soft }} \sim 1$, the waves in case $c$ die out at $k r_{\text {soft }} \sim 0.6$; if the softening length were crucial, the waves in case $c$ should have died away also at $k r_{\text {soft }} \sim 1$. The recurrent behavior of density waves also reveals itself in the evolution of the Toomre $Q$-values discussed below.

In the initial linear regime, case $a$ has a dominant $k_{y}$ at about $k_{y} \lambda_{c} \sim \pi$, which is consistent with the linear stability analysis for the marginally unstable mode. At the later nonlinear stage, the dominant modes are switching between modes $k_{y} \lambda_{c}=\pi$ and $k_{y} \lambda_{c} \sim 2 \pi / 3$. On the other hand, cases $b$ and $c$ have dominant $k_{y}$ at $k_{y} \lambda_{c} \sim \pi$ and $k_{y} \lambda_{c} \sim$ $2 \pi$, respectively, in the linear regime. These dominant wavenumbers coincide with the approximate equality $q^{2}\left(\equiv k_{y}^{2} / k_{d}^{2}\right) \sim 1$. Note from equation (10) that this is the condition where the oscillatory modes, which occur when $q^{2} \leq 1$, become marginally stable. We suspect that these oscillatory modes are suppressed by the Landau damping, which is not included in the above hydrodynamic analysis. 

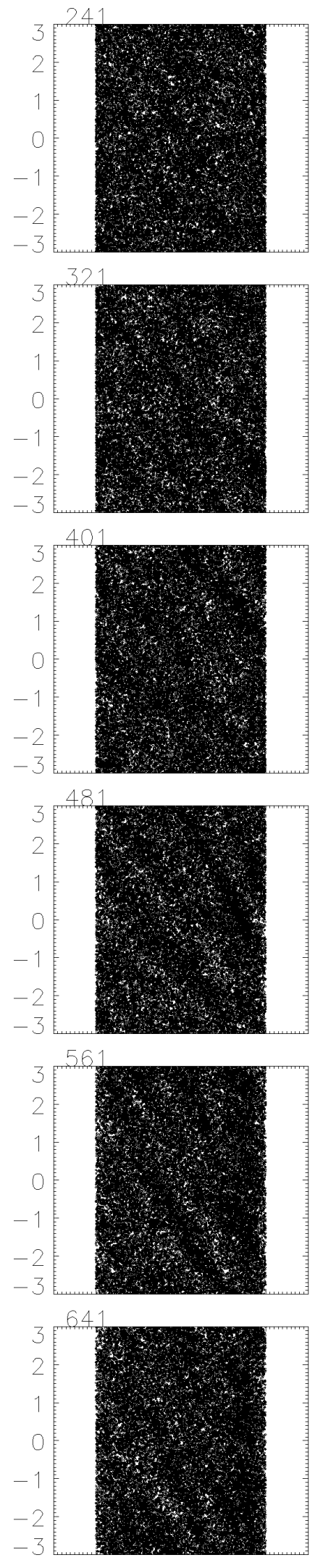
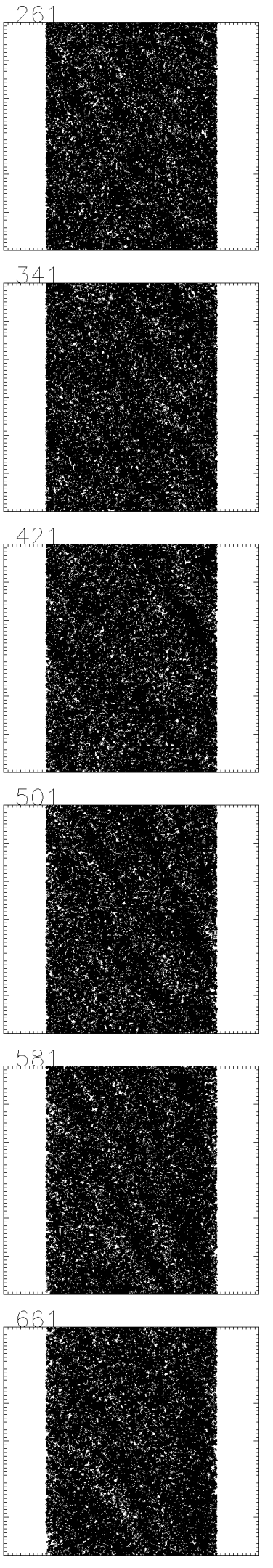
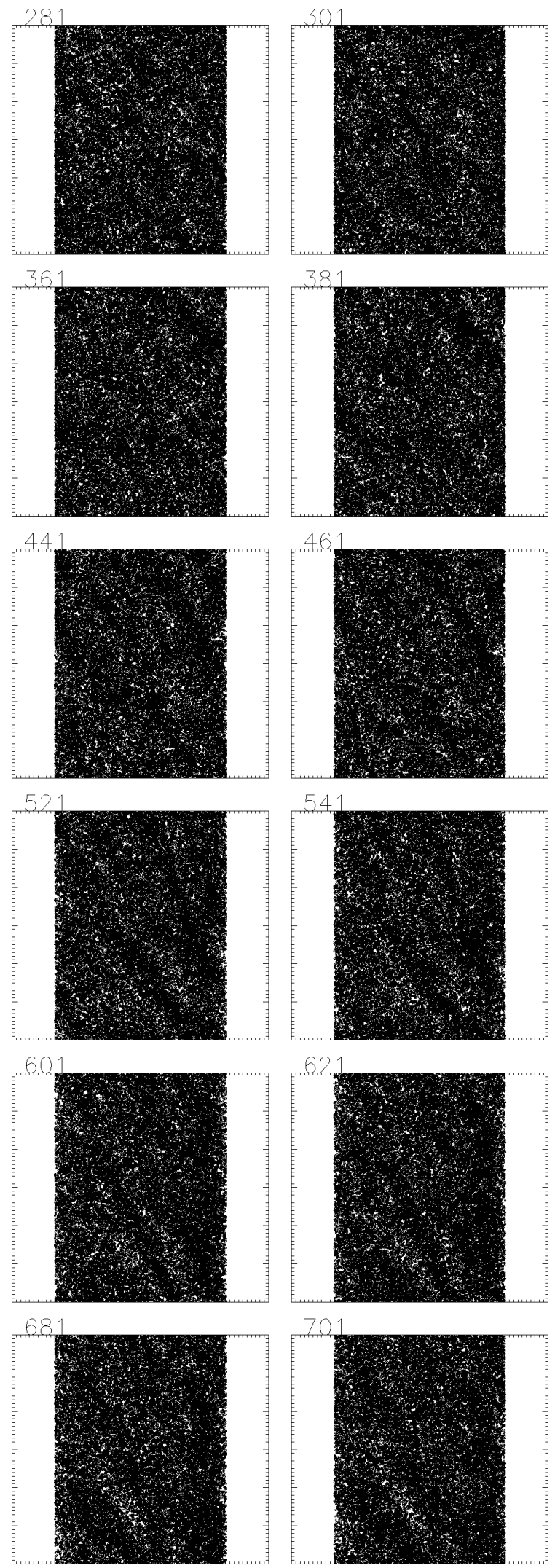

Fig. $1 a$

FIG. 1. - Time sequences of the nonlinear developments for disk density waves in the presence of $(a)$ a rigid halo $\left(k_{d}=0\right),(b)$ a live halo with $k_{d}=\pi / \lambda^{-1}{ }_{c}$, and $(c)$ a live halo with $k_{d}=2 \pi \lambda^{-1}{ }_{c}$. All three cases have the same initial $Q=1.6$ in a periodic shearing box. The particle numbers are 33,600 for $a$ and $b$ but 77,200 for case $c$, the softening lengths chosen are $0.1 \lambda_{c}$ for $a$ and $b$ but $0.05 \lambda_{c}$ for $c$, and the sizes of the shearing box are $4 \lambda_{c} \times 6 \lambda_{c}$ for $a$ and $b$ but $3 \lambda_{c} \times 4 \lambda_{c}$ for $c$. The streamwise $(y)$ direction is along the long side of the box. The frames from upper left to lower right are separated by a fixed time interval $20 \Delta t$, where $628 \Delta t$ corresponds to one rotation. 

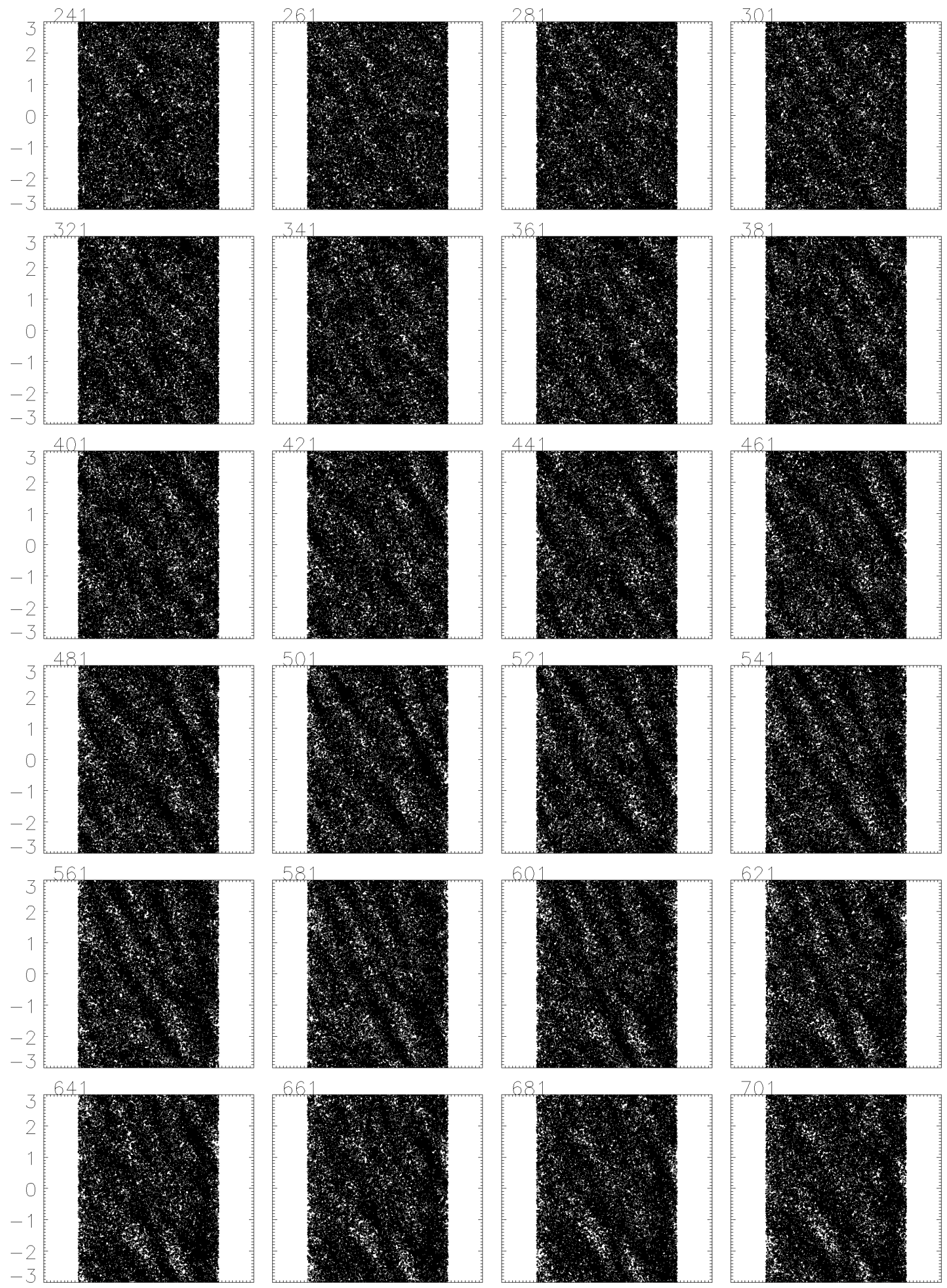

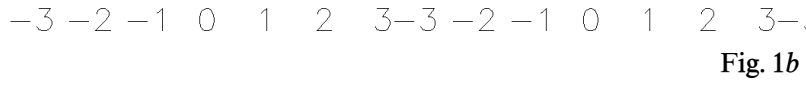



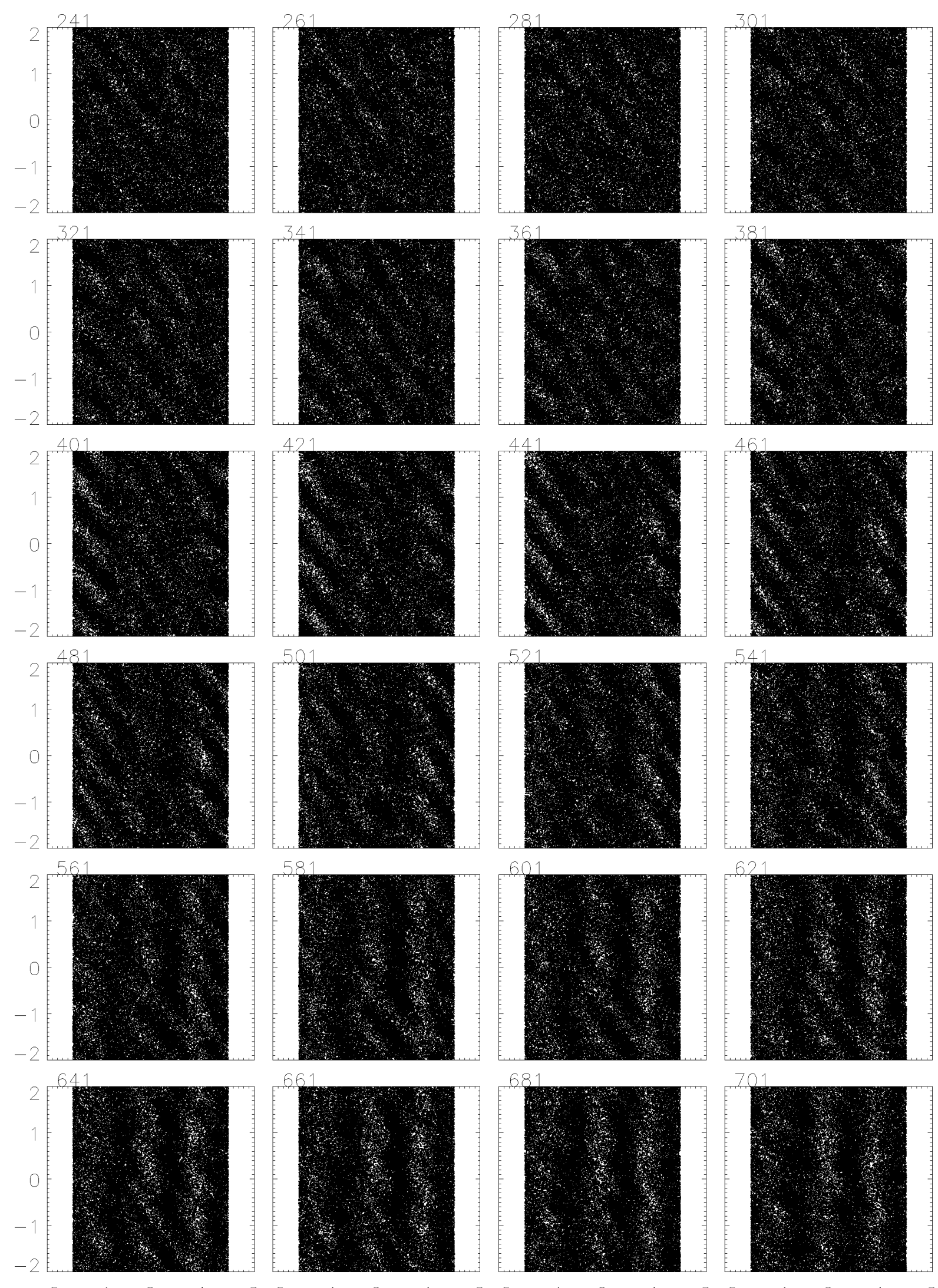

$\begin{array}{lllllllll}-2 & -1 & 0 & 1 & 2-2 & -1 & 0 & 1 & 2- \\ & & & & & & & & \text { Fig. } 1 c\end{array}$ 

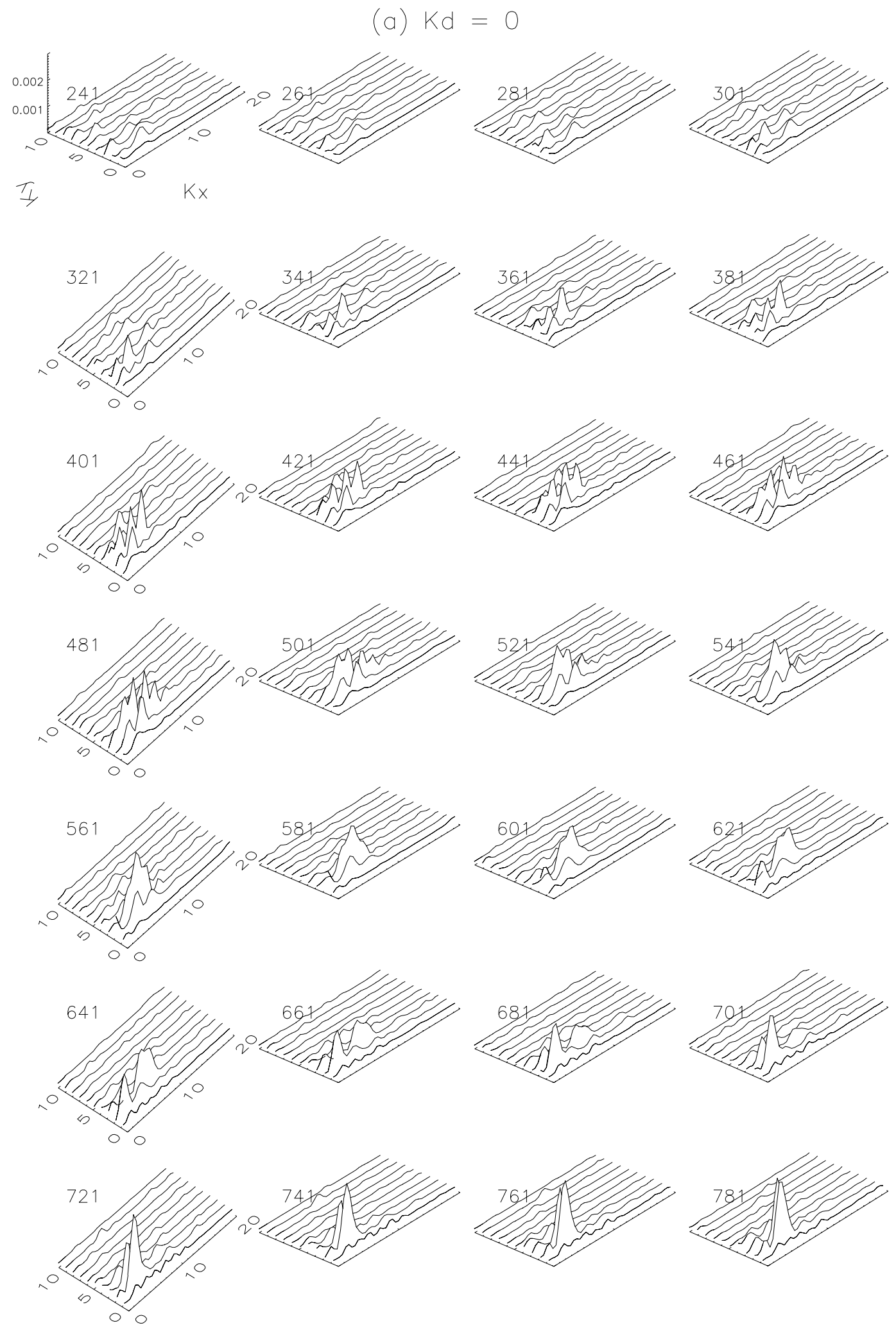

FIG. 2. Corresponding time sequences for the density power spectra $\left|\Sigma\left(k_{x}, k_{y}\right) / \Sigma\right|^{2}$ of Figs. $1 a, 1 b$, and $1 c$

By contrast, the nonoscillatory hydrodynamic modes are strongly unstable near $q^{2} \sim 1$, and they can grow to sizable amplitudes.

In fact, we have deliberately chosen case $b$ such that the condition $q^{2}=1$ corresponds to the most unstable $k_{y}$ of the swing amplification revealed in case $a$. As cases $a$ and $b$ start with exactly the same initial conditions, the effect of swing amplification in the presence of a live halo can be examined by comparing the two cases. Except for the amplitudes, we note the detailed similarity between Figures $2 a$ and $2 b$ in 
No. 1,2000
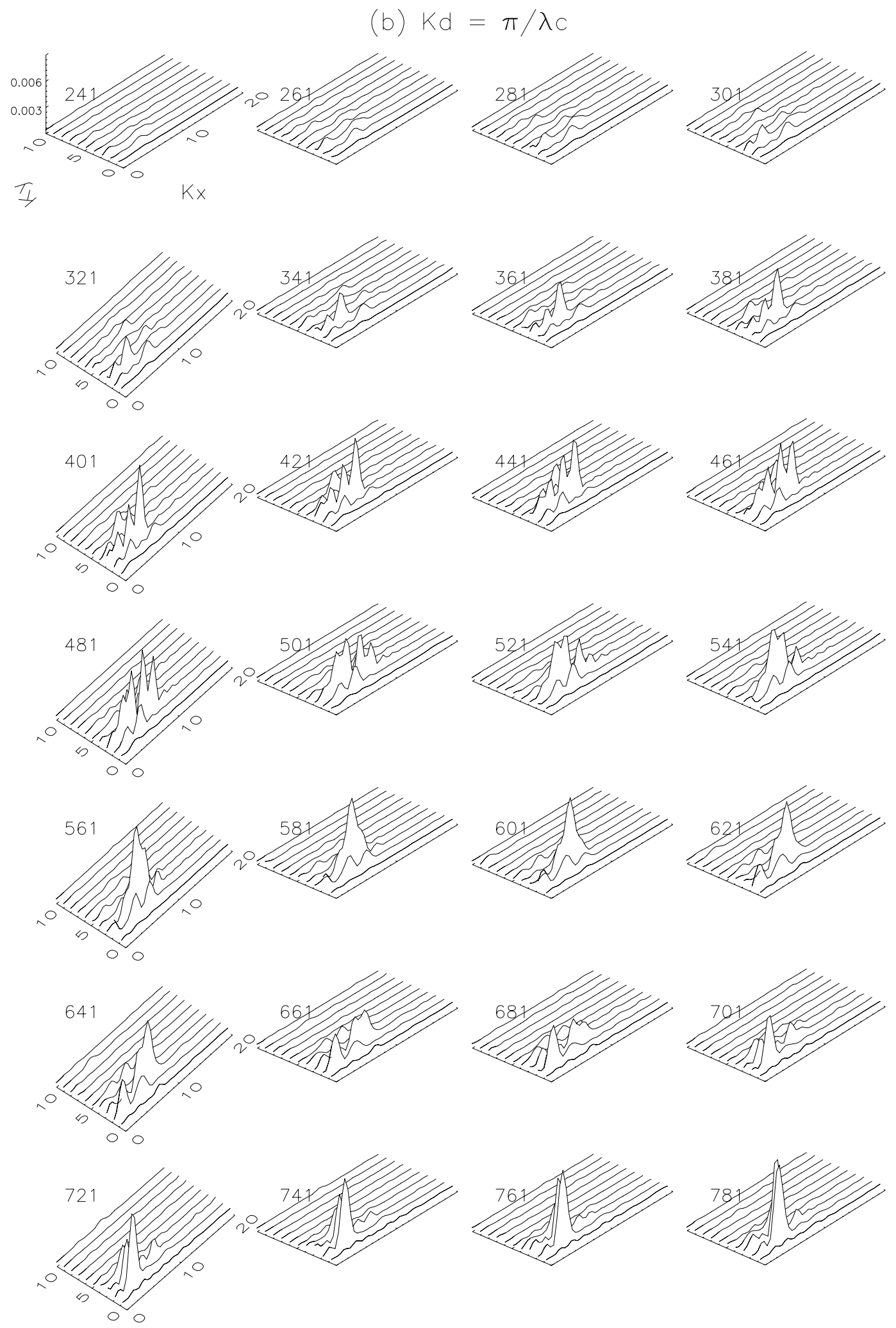

FIG. 2.-Continued

both linear and nonlinear regimes. It implies that the swing amplifier which operates in the rigid halo case is also operative in the weak live halo case.

Saturation of these linearly dominant modes may be caused both by the nonlinear Landau damping, leading to efficient particle heating, and by the nonlinear mode coupling. The damping can be so efficient that the primary modes not only stop growing but also temporarily die out. In the strong live halo case (case $c$ ), nonlinear transfer of modal energy from the $k_{y} \sim(5-6) \lambda_{c}^{-1}$ modes to a low- $k_{y}$ mode, $\left(k_{x}, k_{y}\right) \sim(2 \pi, 1) \lambda_{c}^{-1}$, is apparent after one rotation $(t \sim 628 \Delta t)$ in Figure $2 c$, and this low- $k_{y}$ mode appears to be rather stable throughout the rest of the evolution. Though dying away, these linearly dominant modes can 

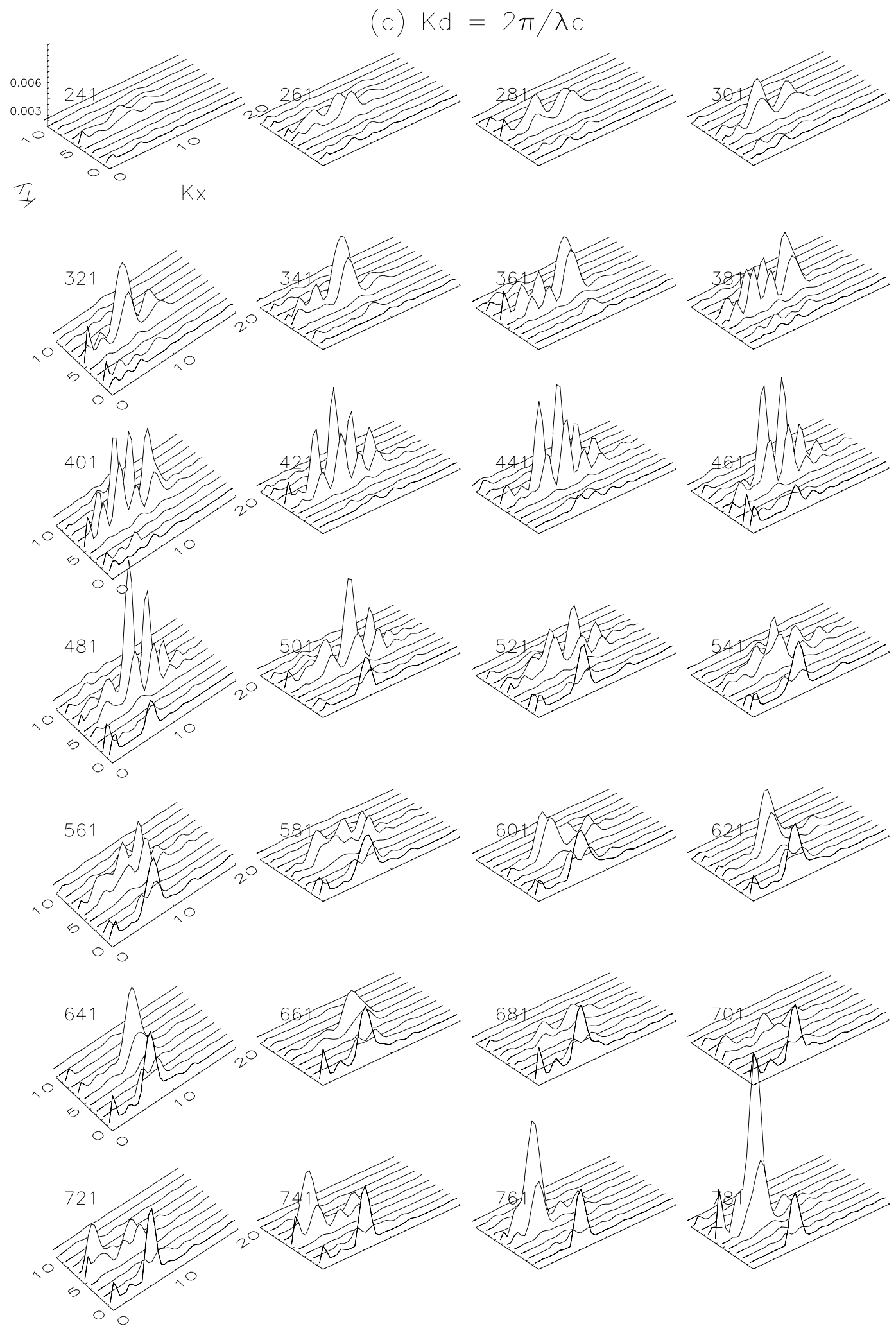

FIG. 2.-Continued

regain their strength and become unstable again. The quasiperiodic recurrence of spiral density waves can be clearly detected in both real space and wavenumber space. Its longterm behavior can also be seen from the oscillatory particleheating curves (Fig. 3), i.e., the evolutionary tracks of $Q$. Comparison of Figures 2 and 3 shows that the heating rates are temporarily large when the linearly dominant modes are dying away and the particles will temporarily give out their energy when the waves are growing.

On average, all $Q$-values in Figure 3 increase with time. Although by the end of our simulations (more than two rotations) the $Q$-values do not appear to saturate for all three cases, the difference in the heating rates is evident. The rigid halo case $(a)$ yields an asymptotic $Q$-value slightly 


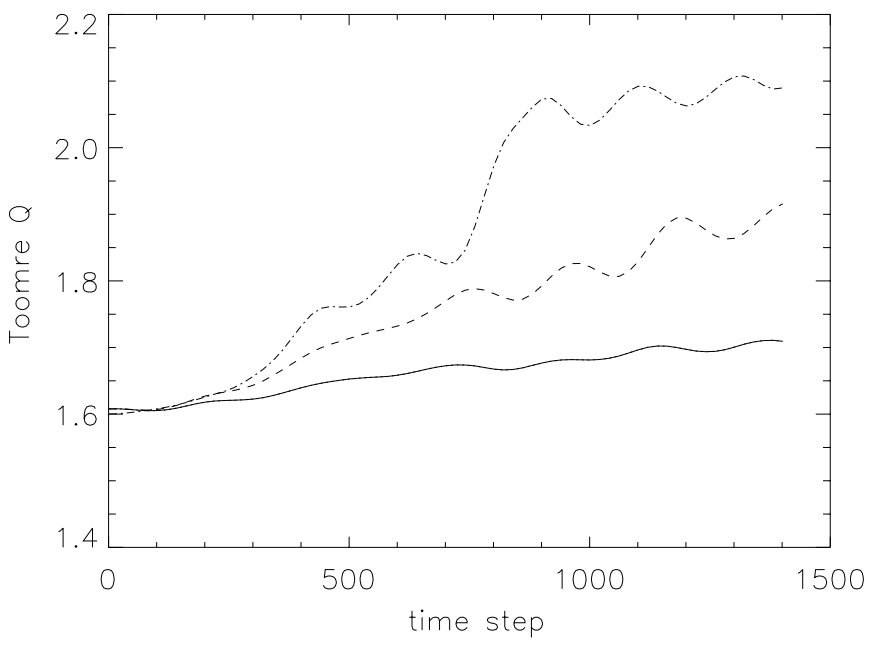

FIG. 3.-Long-term evolution of the Toomre $Q$ for the rigid halo case (solid line) as well as the live halo cases (dashed line for $k_{d} \lambda_{c}=\pi$ and dot-dashed line for $k_{d} \lambda_{c}=2 \pi$ ).

beyond 1.7, whereas the live halo cases yield asymptotic $Q$-values beyond 1.9 and 2.1 for the thick $(b)$ and thin $(c)$ halo cases, respectively.

We may draw a tentative conclusion derived from these numerical results: halo-assisted instabilities can indeed occur. The tendency of the pitch angles in the nonlinear regime is in agreement with the expectation derived from the linear analysis for the gaseous disk; i.e., the more oblate the halo is, the more loosely wound the spiral arms will be. (However, the nonlinear spiral arms are more tightly wound than the linear arms, as a result of shearing by the differential rotation.) In addition, the disk $Q$-value increases with a decreasing halo scale height. Since the pitch angle is an observable quantity and the disk $Q$-value may also be measured independently, these results offer a possibility that one may probe the invisible dark halo scale height $k_{d}^{-1}$ by measuring either of these two quantities. Moreover, as these two quantities can be measured independently, they offer an opportunity for a consistency check in the determination of $k_{d}$. Unfortunately, quantitative determination of the relations between $k_{d}$ and the pitch angle $\psi$ and between $k_{d}$ and the $Q$-value must rely on the simulations of a global diskhalo system. At present, we cannot give any quantitative prediction to be compared with observations in detail.

\section{DISCUSSION AND CONCLUSIONS}

Disk galaxies of high Toomre $Q$ often result from their low surface densities and high angular momentum. Recent studies suggest that LSB galaxies are associated with relatively high angular momentum systems. However, the observations show that low surface brightness galaxies also exhibit spiral arms and some even contain central bulges (McGaugh 1992). Moreover, these galaxies can be spatially isolated, with no evidence of interactions with other galaxies (Mo, et al. 1994). These facts are apparently in contradiction to the expectation that density waves should not be strongly present in the high- $Q$ disks.

The present work proposes a new channel for the excitation of density waves in low surface density galaxies through corotation resonance between disk and halo. The spiral density waves are destabilized only by the part of the halo dark matter that is corotating with the disk. In $\S 2$, we have shown that the spin parameter increases inversely with radius and that in the inner part of the galaxy rotation can indeed contribute to partially supporting the halo against gravity in some high angular momentum galaxies. Moreover, it has also been pointed out that the inner halo can be spun-up (Tremaine \& Ostriker 1999). Therefore, corotation resonance is likely to occur in the inner galaxy for galaxies with relatively high angular momentum. As a contrast, the high surface brightness galaxies are those that have small to medium angular momenta in the halos, and the corotation resonances advocated in the present work are likely not occurring, and their spiral waves are generated by conventional mechanisms. For example, for the average global spin parameter, $\lambda \sim 0.05$, the halo rotation will not be able to satisfy the corotation condition unless the halo core is unrealistically small, e.g., smaller than $0.5 \mathrm{kpc}$.

The underlying physical mechanism for the corotation wave excitation is analogous to the Debye shielding of a charged ion by rapidly moving electrons in a plasma, with, however, an important difference. The gravitational charge is like an imaginary electric charge and hence gives rise to instabilities, as opposed to the stable Langmuir oscillations in a plasma. The rapidly moving halo particles play the same role as the fast electrons in a plasma and the disk stars as the slowly moving ions. In this work, we have presented an analysis in support of such a destabilizing mechanism and numerical results to look into the nonlinear developments. Although the disk can develop into nonlinear regimes as the density waves evolve, the halo has been assumed to still remain in the linear regime. This is because though the potential perturbation $\delta \phi$ can be comparable to the disk velocity dispersion $a^{2}$ in the nonlinear regime, it is still much smaller than $T_{\mathrm{dm}}$, and hence the halo perturbations remain linear, at least during the timescale of the recurrent waves. We note that in this regime the halo column mass is irrelevant to the evolution of disk density waves; rather, it is only the local halo scale height $k_{d}^{-1}$, serving as the imaginary Debye length, that is found to matter. A smaller $k_{d}^{-1}$ yields a more unstable spiral wave, which has a larger pitch angle and which drives the disk toward a higher value of $Q$. Of course, on a longer timescale the halo has to adjust, and the more massive the halo is the longer this timescale will be. However, this important aspect has been excluded from the present consideration.

We now return to comment on the disk-halo corotation, which is valid, as shown in the Appendix, when the difference in the rotation speeds of disk and halo is smaller than the halo velocity dispersion. The disk-halo corotation is generally expected to hold only within a finite part of the disk. In the noncorotating region the difference of the rotation speeds is supersonic (with respect to the halo particles), and the instability studied in this work should be suppressed since the medium is moving too fast for a positivefeedback chain to establish. Realistically, the unstable waves excited in the corotating region should leak into the noncorotating region and get partially stabilized. The wave angular momentum should also be transferred to the halo, but how this proceeds is beyond the scope of this work. Several recent works have focused on this issue of angular momentum transfer, but in a different context (Combes et al. 1990; Raha et al. 1991; Little \& Calberg 1991; Hernquist \& Weinberg 1992; Debattista \& Sellwood 1998, 1999; Tremaine \& Ostriker 1999). Therefore, our simplified local model aims simply to demonstrate the plausibility of a new 
excitation mechanism for the disk spiral density waves in a high Toomre $Q$ disk. The present local results are still far from being able to compare with observations quantitatively.
This work is supported in part by the National Science Council of Taiwan under grants NSC88-2112-M-008-039 and NSC88-2112-M-008-040.

\section{APPENDIX}

\section{CRITERION FOR THE ADIABATIC HALO RESPONSE OF A COROTATING HALO}

For simplicity we consider a local three-dimensional shearing box for a galactic halo near a galactic disk. Define $f \equiv$ $T_{\mathrm{dm}} \ln \left(\rho_{\mathrm{dm}}\right)+\phi$; the quantity $f$ measures the deviation from the adiabatic halo response. The linearized momentum equation thus reads

$$
-i \sigma u-2 \Omega_{\mathrm{dm}} v=-\frac{\partial \delta f}{\partial x}, \quad-i \sigma v+2 B_{\mathrm{dm}} u=-i k_{y} \delta f, \quad-i \sigma w=-\frac{\partial \delta f}{\partial z},
$$

where $u, v$, and $w$ are the $x$-, $y$-, and $z$-components of velocity perturbations, respectively; the subscripts $x$ and $z$ of $\delta f$ denote the spatial derivatives, respectively; and Fourier transformation in $y$ and $t$ has been conducted, with $\sigma \equiv \omega+k_{y} A_{\mathrm{dm}} x$ and $\omega$ being the frequency. One may solve for $u$ and $v$ in favor of $\delta f$ and $\delta f_{x}$ from the $x$ - and $y$-components of equation (A1). When substituting $u, v$, and $w$ into the perturbed continuity equation

$$
i \sigma \delta \rho_{\mathrm{dm}}=\frac{\partial}{\partial z}\left(\rho_{\mathrm{dm}} w\right)+\rho_{\mathrm{dm}}\left(\frac{\partial u}{\partial x}+i k_{y} v\right)
$$

we obtain

$$
\begin{gathered}
4 \pi G \delta \rho_{\mathrm{disk}}-\left(\frac{\partial^{2}}{\partial x^{2}}+\frac{\partial^{2}}{\partial z^{2}}-k_{y}^{2}\right) \delta \phi \\
=\frac{4 \pi G}{\sigma^{2}} \frac{\partial}{\partial z}\left(\rho_{\mathrm{dm}} \delta f_{z}\right)+\frac{4 \pi G \rho_{\mathrm{dm}}}{\sigma}\left[\frac{\partial}{\partial x}\left(\frac{\sigma \delta f_{x}-2 k_{y} \Omega_{\mathrm{dm}} \delta f}{\kappa_{\mathrm{dm}}^{2}-\sigma^{2}}\right)+k_{y} \frac{2 B_{\mathrm{dm}} \delta f_{x}-k_{y} \sigma \delta f}{\kappa_{\mathrm{dm}}^{2}-\sigma^{2}}\right],
\end{gathered}
$$

where the Poisson's equation has been used and $\delta \rho_{\text {disk }}$ denotes the density perturbation of the disk, which is concentrated at $z=0$. The two terms on the right of equation (A3) come from the corresponding terms on the right of equation (A2).

Below, we present an order of magnitude estimate, which will be followed by a quantitative analysis. Now, compare the two terms on the right of equation (A3). For a strictly corotating halo, we find that $|\sigma|$ is much smaller than $\Omega$ in the relatively weak growing phase of wave instability discussed in this work. It follows that the first term is much greater than the second term. That is, the halo density response results almost entirely from vertical compression or rarefaction, which justifies equation (6). Next, since the first term on the left exists only on the disk, above the disk the second term on the left must balance the first term on the right. As the coefficient of the latter is so large, we must demand

$$
\frac{\partial}{\partial z}\left(\rho_{\mathrm{dm}} \frac{\partial \delta f}{\partial z}\right) \rightarrow 0
$$

which leads to a unique solution, i.e., $\delta f \rightarrow 0$, since $\delta f$ is an even function of $z$ for the density wave. This result leads to equation (5), the adiabatic halo response. An odd solution can also be obtained, i.e., $\partial \delta f / \partial z \propto \rho_{\mathrm{dm}}^{-1}$, and it leads to the vertical bending of the disk. The bending waves are excluded from the consideration of this work but studied in separate works (Tseng 2000; Chiueh, Wu, \& Tseng 2000).

However, the halo and disk may not corotate, differing by a finite angular frequency $\Delta \Omega$. We now have $|\sigma| \sim \Delta \Omega$ in the unstable phase of the disk density wave. The last paragraph has shown that as long as $\Delta \Omega^{2} \ll \Omega^{2}$, we may justify the adiabatic approximation of the halo response. In fact, for the halo to be in equilibrium, we must also have $4 \pi G \rho_{\mathrm{dm}}(R) \sim 2 T_{\mathrm{dm}} / R^{2}$ if the halo is approximately isothermal; on the other hand, for the disk to be in equilibrium within the halo potential, we must also demand $4 \pi G \rho(R) \sim 2 \Omega^{2}$. Therefore, the validity of the adiabatic halo response amounts to the validity of the inequality

$$
R^{2} \Delta \Omega^{2} / 2 T_{\mathrm{dm}} \ll 1
$$

or

$$
\Delta \Omega^{2} / 4 \pi G \rho_{\mathrm{dm}} \ll 1
$$

near the galactic disk.

The above approximate analysis can be put in a more rigorous framework. We proceed by seeking the possible separation of variables for equation (A3). Following the method introduced in our previous work for an accretion-disk problem (Chiueh \& Tseng 1994), we let

$$
\frac{\partial}{\partial x}\left[\frac{\sigma(\partial \delta f / \partial x)-2 k_{y} \Omega_{\mathrm{dm}} \delta f}{\kappa_{\mathrm{dm}}^{2}-\sigma^{2}}\right]+k_{y} \frac{2 B_{\mathrm{dm}}(\partial \delta f / \partial x)-k_{y} \sigma \delta f}{\kappa_{\mathrm{dm}}^{2}-\sigma^{2}}=-\frac{a \delta f}{\sigma}
$$


and

$$
\frac{\partial}{\partial z}\left(\rho_{\mathrm{dm}} \frac{\partial \delta f}{\partial z}\right)=b \rho_{\mathrm{dm}} \delta f,
$$

where $a$ and $b$ are approximated as the eigenvalues, and they have dimensions as the squared wavenumber and magnitudes about the squared horizontal and vertical wavenumbers, respectively. Equation (A3) thus becomes

$$
\left[\frac{\partial^{2}}{\partial x^{2}}+\frac{\partial^{2}}{\partial z^{2}}-k_{y}^{2}+\frac{4 \pi G \rho_{\mathrm{dm}}(a+b)}{\sigma^{2}+(a+b) T_{\mathrm{dm}}}\right] \delta \phi=4 \pi G \delta \rho_{\text {disk }} .
$$

When $\sigma^{2} /(a+b) T_{\mathrm{dm}} \ll 1$, we recover equation (7), the modified form of Poisson's equation. Since $(a+b)$ has a magnitude on the order of or even larger than $R^{-2}$, it follows that $R^{2} \Delta \Omega^{2} / 2 T_{\mathrm{dm}} \ll 1$ is indeed the criterion for the validity of the adiabatic halo response.

Barnes, J., \& Efstathiou, G. 1987, ApJ, 319, 575

Binney, J., Jiang, I. G., \& Dutta, S. 1998, MNRAS, 297, 1237

Binney, J., \& Tremaine, S. 1987, Galactic Dynamics (Princeton: Princeton Univ. Press)

Bouwens, R., \& Silk, J. 2000, ApJ, in press (astro-ph/0002133)

Catelan, P., \& Theuns, T. 1997, MNRAS, 292, 225

Chiueh, T., \& Tseng, Y. H. 1994, ApJ, 435, 379

Chiueh, T., Wu, J. M., \& Tseng, Y. H. 2000, Chinese J. Phys., 38, 120

Cole, S., \& Lacey, C. 1996, MNRAS, 281, 716

Combes, F., Debbasch, F., Friedli, D., \& Pfenniger, D. 1990, A\&A, 233, 82

Dalcanton, J. J., \& Bernstein, R. A. 2000, in ASP Conf. Ser. 197, Dynamics of Galaxies: From the Early Universe to the Present, ed. F. Combes,

G. A. Mamon, \& V. Charmandaris (San Francisco: ASP), 161

Dalcanton, J. J., Spergel, D. N., \& Summers, F. J. 1997, ApJ, 482, 659

Debattista, V. P., \& Sellwood, J. A. 1998, ApJ, 493, L5

. 1999, ApJ, 513, L107

Goldreich, P., \& Lynden-Bell, D. 1965, MNRAS, 130, 125

Goldreich, P., \& Tremaine, S. 1978, ApJ, 222, 850

Hausman, M. A., \& Ostriker, J. P. 1978, ApJ, 224, 320

Heavens, A. F. \& Peacock, J. A. 1988, MNRAS, 232, 339

Hernquist, L., \& Weinberg, M. D. 1992, ApJ, 400, 80

Heyl, J. S., Hernquist, L., \& Spergel, D. N. 1996, ApJ, 463, 69

Jiang, I.-G., \& Binney, J. 1999, MNRAS, 303, L7

Jimenez, R., Heaven, A. F., Hawkins, M. R. S., \& Padoan, P. 1997, MNRAS, 292, L5

Julian, W. H., \& Toomre, A. 1966, ApJ, 146, 810

Little, B., \& Calberg, R. G. 1991, MNRAS, 250, 161

Mao, S., \& Mo, H. J. 1998, MNRAS, 296, 847

McGaugh, S. S. 1992, Ph.D. thesis, Univ. Michigan

\section{REFERENCES}

Mo, H. J., Mao, S., \& White, S. D. M. 1998, MNRAS, 295, 319

Mo, H. J., McGaugh, S. S., \& Bothun, G. D. 1994, MNRAS, 267, 129

Morozov, A. G. 1980, Soviet Astron., 24, 391

Nagashima, M., \& Gouda, N. 1998, MNRAS, 301, 849

Navarro, J. F., Frenk, C. S., \& White, S. D. M. 1995, MNRAS, 275, 720 1997, ApJ, 490, 493

Olling, R. P. 1995, AJ, 110, 591 .1996, AJ, 112, 481

O'Nell, K., Bothun, G., \& Cornell, M. 1997, AJ, 113, 1212

Peebles, P. J. E. 1969, ApJ, 155, 393

Raha, N., Sellwood, J. A., James, R. A., \& Kahn, F. D. 1991, Nature, 352, 411

Sackett, P. D., Rix, H.-W., Jarvis, B. J., \& Freeman, K. C. 1994, ApJ, 436, 629

Sackett, P. D., \& Sparke, L. S. 1990, ApJ, 361, 408

Schneider, S. E., \& Schombert, J. M. 2000, ApJ, 530, 286

Steinmetz, M., \& Bartelmann, M. 1995, MNRAS, 272, 570

Swaters, R. A., Madore, B. E., \& Trewhella, M. 2000, ApJ, 531, L107

Toomre, A. 1981, in The Structure and Evolution of Normal Galaxies, ed.

S. M. Fall \& D. Lynden-Bell (Cambridge: Cambridge Univ. Press), 111

Tremaine, S., \& Ostriker, J. P. 1999, MNRAS, 306, 662

Tseng, Y. H. 2000, Chinese J. Phys., 38, 111

van den Bosch, F. C., Robertson, B. E., Dalcanton, J. J., \& de Blok, W. J. G. 2000, AJ, 119, 1579

Warren, M. S., Quinn, P. J., Salmon, J. K., \& Zurek, W. H. 1992, ApJ, 399, 405

White, S. D. M. 1984, ApJ, 286, 38

Yue, Z. Y. 1982, Geophys. Astrophys. Fluid Dyn., 20, 1 